\title{
Localized lymphoid hyperplasia of the spleen: a rare benign condition grossly mimicking malignancy
}

\author{
Jennifer A. Muir ${ }^{1} \cdot$ Marites Espinas $^{2} \cdot$ Jan Delabie $^{1}$
}

Received: 19 August 2015 / Accepted: 2 December 2015 /Published online: 14 December 2015

(C) Springer-Verlag Berlin Heidelberg 2015

\begin{abstract}
Lymphoid hyperplasia of the splenic white pulp typically entails a diffuse expansion of white pulp throughout the spleen. However, rarely, lymphoid hyperplasia may manifest as one or more localized nodules. This localized form of lymphoid hyperplasia was first described in 1983, and since then, there have been no further reports in the literature. We report one additional case which was initially clinically suspected to represent metastatic malignancy. The patient was a healthy 53-year-old woman who presented with symptoms of biliary colic and underwent laparoscopic cholecystectomy. During surgery, several white-tan nodules were noted on the spleen. Diagnostic splenectomy was ultimately performed, and histologic examination and immunohistochemical characterization of the nodules revealed wellcircumscribed aggregates of secondary lymphoid follicles embedded in a T cell-rich interfollicular zone, much like tertiary nodules seen in lymph nodes and unlike the normal microanatomy of the white pulp. Based on these findings, localized lymphoid hyperplasia (LLH) of the spleen was diagnosed. The aetiology of LLH is unknown, though it is speculated to represent a localized response to antigenic stimulation akin to florid reactive hyperplasia in a solitary lymph node. It is interesting that, despite the now widespread use of highresolution abdominal imaging, LLH remains a rare diagnosis.
\end{abstract}

Keywords Spleen $\cdot$ Lymphoid hyperplasia $\cdot$ Case report

Jennifer A. Muir

jennifer.muir@mail.utoronto.ca

1 Laboratory Medicine Program, University Health Network, University of Toronto, Faculty of Medicine, 200 Elizabeth St., Toronto, ON M5G 2C4, Canada

2 Department of Pathology, Lakeridge Health, Oshawa, ON, Canada

\section{Introduction}

Reactive lymphoid hyperplasia of the splenic white pulp is common, occurring in a variety of infectious, inflammatory, and autoimmune conditions. Typically, reactive hyperplasia entails a diffuse expansion of the white pulp in a follicular or non-follicular pattern, in which the basic microanatomy of the white pulp with periarteriolar sheath and marginal zone is maintained [1]. However, rarely, the spleen may show a localized hyperplasia consisting of well-circumscribed lymphoid aggregates that do not resemble the normal white pulp microanatomy. This phenomenon was first reported by Burke and Osborne in 1983 [2], and to our knowledge, has not been described in the literature since then. It is important to recognize this entity since it is a benign condition whose gross appearance may mimic several other diseases, including localized splenic involvement by lymphoma or metastatic malignancy. Herein, we report a case of localized lymphoid hyperplasia (LLH) of the spleen that was initially clinically suspected to represent metastatic malignancy.

\section{Clinical history}

The patient is a generally healthy 53-year-old woman with no significant past medical history other than having been a former smoker. She presented with symptoms of biliary colic and underwent laparoscopic cholecystectomy. At the time of the surgery, several pale, tan nodules were noted on the surface of the spleen. Post-operative CT imaging confirmed multiple nodules scattered throughout the splenic parenchyma.

The patient was completely asymptomatic, and other investigations were all within normal limits, including complete blood counts and a bone marrow biopsy. Lymphoma and metastatic malignancy were high on the clinical differential 
diagnosis, but the patient had no history of prior primary malignancy and no other suspicious findings on clinical examination or abdominal imaging, including no apparent lymphadenopathy. Furthermore, she had recently undergone a normal screening colonoscopy and normal screening mammogram. The resected gallbladder showed benign changes only. Given the patient's smoking history, a CT scan of the chest was performed; no evidence of a primary malignancy was found.

A follow-up abdominal ultrasound demonstrated a stable spleen size with no splenomegaly, but did show an increase in the number of parenchymal lesions. Given the lack of a clear diagnosis and the increase in the number of splenic nodules, the decision was made to perform a diagnostic laparoscopicassisted splenectomy.

\section{Materials and methods}

Haematoxylin and eosin (H\&E)-stained sections were prepared from formalin-fixed, paraffin-embedded tissue. Immunohistochemistry was performed on formalin-fixed, paraffin-embedded tissue using commercially available antibodies with appropriate on-slide controls.

\section{Results}

Grossly, the spleen was of normal size and shape, weighing 125 g. A slightly exophytic, pink-tan nodule measuring $0.4 \times$ $0.3 \times 0.3 \mathrm{~cm}$ was noted on the capsular surface, $1.5 \mathrm{~cm}$ from the hilar margin. Serial sectioning of the spleen revealed multiple other pink-tan, well-circumscribed nodules within the splenic parenchyma, ranging from 0.1 to $0.5 \mathrm{~cm}$ in greatest dimension (Fig. 1). The background parenchyma was grossly unremarkable.

Histologically, the pink-tan nodules seen grossly corresponded to well-circumscribed nodules of lymphoid cells scattered throughout the spleen, having the appearance of tertiary nodules commonly seen in lymph nodes (Fig. 2a-b). The nodules were comprised of multiple secondary lymphoid follicles arranged around a nodular extrafollicular area containing small lymphocytes, immunoblasts, plasmacytoid cells, and plasma cells. The secondary follicles varied somewhat in size and shape, had normal mantle and marginal zones, and had germinal centres with tingible body macrophages and typical light and dark zones. The nodules displayed no cytologic atypia, and the remainder of the red and white pulp was normal. There were very few secondary follicles in the white pulp.

Immunohistochemistry further illustrated the structure of the nodules (Fig. 2c-m). Multiple secondary follicles were present, each displaying CD20-positive, CD10-positive, and BCL-2-negative germinal centre cells. CD3 highlighted the T

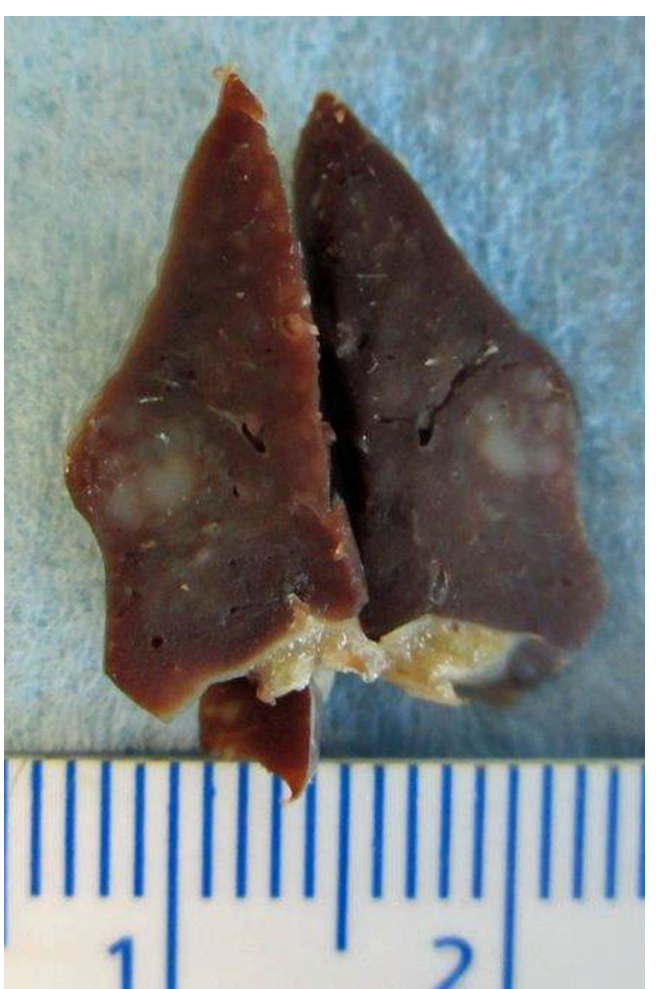

Fig. 1 Gross appearance of localized lymphoid hyperplasia of the spleen. The spleen was of normal size and shape, weighing $125 \mathrm{~g}$. Wellcircumscribed, tan nodules are visible within the splenic parenchyma

cell-rich extrafollicular zone between secondary follicles. Most T cells were CD4-positive with scattered CD8positive cells also present. CD8 is also known to highlight splenic sinusoids, and in this case demonstrated well-formed sinusoids in the red pulp and the absence of sinusoids within the nodules. CD23 demonstrated a normal follicular dendritic cell network and highlighted scattered $\mathrm{B}$ cells in the mantle zone. $\mathrm{IgD}$ was positive in the mantle zone, and BCL-2 was positive in mantle and marginal zone $\mathrm{B}$ cells as well as T cells in the interfollicular zone. Interdigitating dendritic cells with a stellate morphology were seen in the extrafollicular zone and were highlighted by S100. MIB-1 staining showed polarization of the germinal centres of some follicles.

On the basis of this morphology and the corresponding immunohistochemical profile, a diagnosis of localized lymphoid hyperplasia of the spleen was rendered.

\section{Discussion}

Localized lymphoid hyperplasia (LLH) of the spleen is a rare condition which was first described in 1983 by Burke \& Osborne [2]. To our knowledge, since their original description, no further cases have been reported in the literature. In this paper, we describe an additional case of LLH which was clinically suspected to represent metastatic malignancy. 

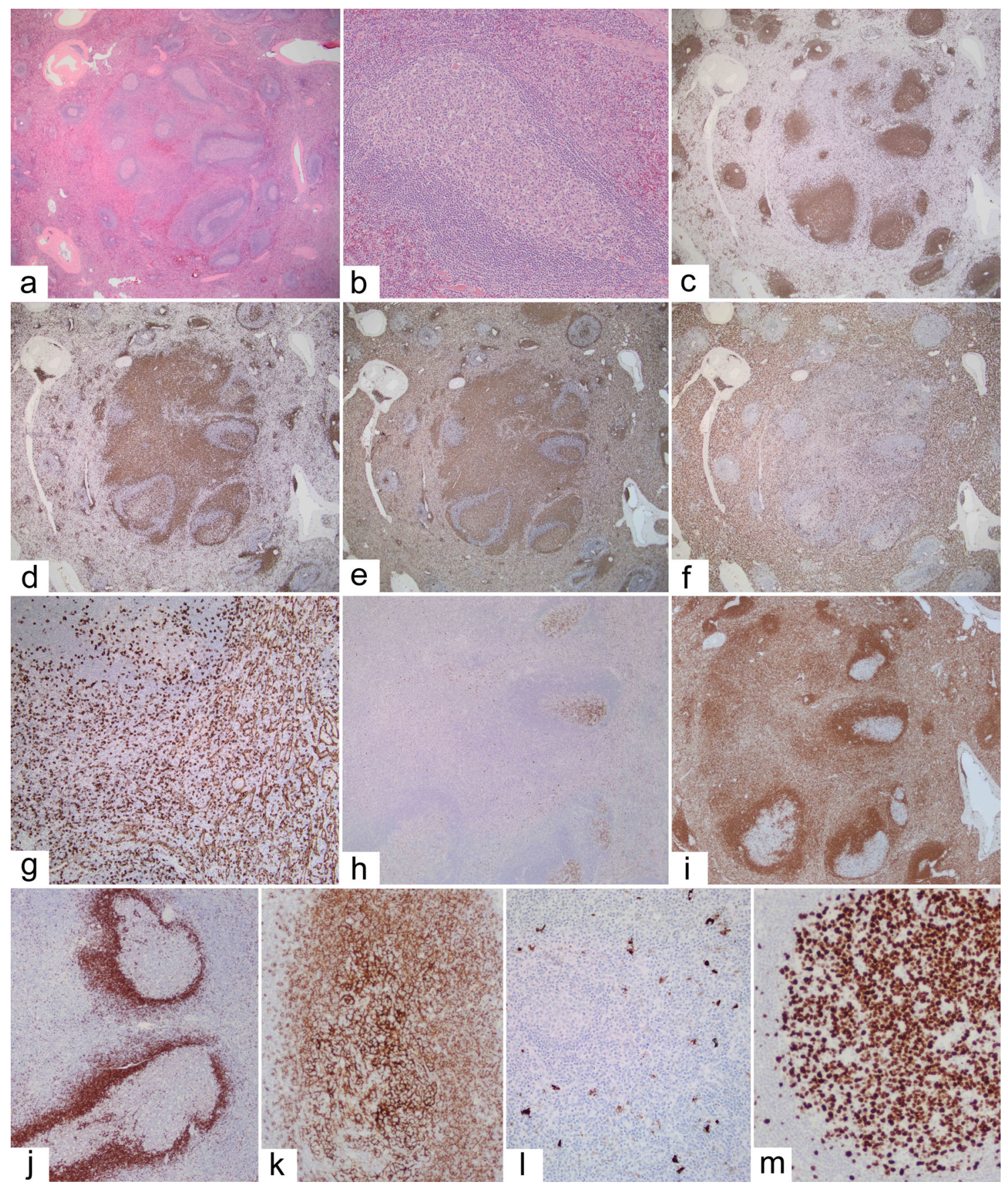

Fig. 2 Microscopic appearance of localized lymphoid hyperplasia of the spleen. a Multiple secondary follicles aggregate together to form a localized nodule (haematoxylin and eosin $(\mathrm{H} \& \mathrm{E}), 20 \times$ ). b A higher power view of one of the follicles shows normal mantle and marginal zones and a normal germinal centre with dark and light zones and tingible body macrophages $(\mathrm{H} \& \mathrm{E}, 100 \times)$. c CD20 immunohistochemistry highlights B cells within follicles $(20 \times)$. d The extrafollicular zone is rich in CD3-positive T cells (20×). Most T cells are CD4-positive (e) with some CD8-positive cells (f). g CD8 also highlights splenic sinusoids, demonstrating well-formed sinusoids in the red pulp and the

absence of sinusoids within the nodules $(100 \times)$. h CD10 highlights germinal centre B cells $(40 \times)$. i BCL- 2 is positive in the mantle and marginal zones of follicles and in $\mathrm{T}$ cells in the extrafollicular zone $(25 \times)$. $\mathbf{j}$ IgD highlights the mantle zones $(40 \times)$. $\mathbf{k}$ CD23 is positive in the follicular dendritic cell network of a follicle as well as some mantle zone B cells $(100 \times)$. I Interdigitating dendritic cells with a stellate morphology were seen in the extrafollicular zone and were highlighted by S100 $(200 \times)$. $\mathbf{m}$ There is polarization of some germinal centres on MIB-1 staining $(150 \times)$

Grossly, LLH presents as one or more tan-white, fleshy, circumscribed lesions within the spleen. This appearance carries a wide differential diagnosis, including infection (e.g.

tuberculosis, histoplasmosis), inflammatory conditions (e.g. sarcoidosis), primary neoplasia (e.g. splenic hamartoma, isolated splenic lymphoma), or metastatic carcinoma, lymphoma, 
or melanoma [3-5]. In LLH, as in many of these conditions, splenic lesions are typically detected incidentally. In their initial report, Burke \& Osborne described a series of eight cases in which solitary nodules were noted in the spleen following splenectomy for staging of known lymphoma ( $7 / 8$ cases) or for management of autoimmune haemolytic anaemia (1/8) [2]. Patient ages ranged from 15 to 84 , and all but one spleen contained one or more grossly identifiable, round-to-ovoid, grey-white, fleshy nodules ranging in size from 0.1 to $1.0 \mathrm{~cm}$. One spleen did not have any gross abnormality, but a nodule of LLH was found on histologic examination.

Microscopic examination of nodules in LLH reveals localized, well-circumscribed aggregates of lymphoid cells. The nodules are often comprised of clusters of benign secondary follicles with an intervening zone of predominantly CD4positive $\mathrm{T}$ cells, as demonstrated in the case we present [2]. Other cases may show a localized, nodular proliferation of plasma cells or immunoblasts without formation of germinal centres [2]. In cases with germinal centre formation, the architecture of the lymphoid tissue resembles the tertiary nodules commonly observed in reactive lymph nodes, but which is not seen in normal splenic white pulp [6]. Of interest, this lymphoid tissue is not arranged around a central arteriole the way normal white pulp is.

It is the localized nature of the lymphoid aggregates in LLH and their tendency to form only one or a few macroscopically visible nodules that distinguish LLH from the reactive lymphoid hyperplasia usually seen in the spleen. In normal spleens, lymphoid cells are found in the white pulp, which is distributed throughout the spleen around arterioles and functions to initiate immune responses to circulating antigens [7]. The normal white pulp may become diffusely expanded in autoimmune conditions such as rheumatoid arthritis (Felty syndrome), in cytopenic conditions such as thrombotic thrombocytopenic purpura (TTP), immune thrombocytopenic purpura (ITP), and acquired haemolytic anaemia, in viral infections including HIV/AIDS, in other conditions such as graft rejection and Castleman's disease, or in response to an idiopathic antigenic stimulation [1]. Macroscopically visible lesions may be formed, but these lesions are multiple, and unlike the lesions of LLH, they are uniform and evenly distributed throughout the spleen. Furthermore, diffuse reactive lymphoid hyperplasia often results in splenomegaly, which is not seen in LLH.

Next, it is important to distinguish LLH from splenic involvement by lymphoma. Morphology on haematoxylin and eosin (H\&E)-stained sections is the most important means of evaluation, and usually LLH is readily distinguished as a benign process on the basis of its classic reactive secondary follicles, which do not encroach on one another, retain light and dark zones and tingible body macrophages in their germinal centres, and have normal mantle zones. The typical immunohistochemical staining pattern is described above and mirrors that seen in a reactive lymph node. Conversely, as noted by Burke and Osborne, the diagnosis of LLH lacking germinal centre formation can be more challenging, as the proliferating immunoblasts seen in that form of LLH can resemble Hodgkin or Reed-Sternberg cells [2]. However, close examination should reveal single, eccentric, basophilic nucleoli characteristic of immunoblasts, and the absence of the cellular background of Hodgkin's disease. Whilst immunoblasts may be positive for CD30, they should be negative for CD15.

The aetiology of LLH is unknown. Burke and Osborne speculated that the lesions might be an unusual, localized response to antigenic stimulation, much akin to florid reactive hyperplasia in a solitary lymph node [2]. Other evidences of antigenic stimulation in their series included six cases with mild background diffuse follicular hyperplasia of the white pulp, two cases with epithelioid granulomas, and one case being from a patient with autoimmune haemolytic anaemia [2]. In our case, there was no supporting evidence for antigenic stimulation, and our patient had no known history or clinical symptoms of any autoimmune disease. It is possible that our patient had a subclinical infection causing an unusual localized response or an early/asymptomatic autoimmune process. However, the precise aetiology and pathophysiology of LLH remain obscure.

Finally, the rarity of LLH, its asymptomatic presentation, and the decrease in the number of splenectomy specimens now examined due to the discontinuation of staging splenectomy in lymphoma are all possible reasons for the dearth of published literature surrounding LLH. Indeed, 7/8 cases diagnosed by Burke and Osborne over a 7-year period were found at the time of staging splenectomy. However, with the increasing use of high-resolution abdominal imaging for investigation and follow-up of other conditions, one might expect a parallel increase in incidentally discovered splenic lesions such as LLH, which, as demonstrated in our case, can produce visible lesions on CT and ultrasound. It is interesting that, despite the now widespread use of such imaging, LLH remains an extremely rare diagnosis today.

Compliance with ethical standards This article does not contain any studies with human participants or animals performed by any of the authors.

Conflict of interest The authors declare that they have no competing interests.

\section{References}

1. Burke JS (1981) Surgical pathology of the spleen: an approach to the differential diagnosis of splenic lymphomas and leukemias. Part I. Diseases of the white pulp. Am J Surg Pathol 5:551-563

2. Burke JS, Osborne BM (1983) Localized reactive lymphoid hyperplasia of the spleen simulating malignant lymphoma. A report of seven cases. Am J Surg Pathol 7:373-380 
3. Warshauer DM, Hall HL (2006) Solitary splenic lesions. Semin Ultrasound CT MR 27:370-388

4. Pejic RN, Lee DT (2004) Incidental splenic lesions. J Fam Pract 53: 456-460

5. Kamaya A, Weinstein S, Desser TS (2006) Multiple lesions of the spleen: differential diagnosis of cystic and solid lesions. Semin Ultrasound CT MR 27:389-403
6. van den Oord JJ, de Wolf-Peeters C, Desmet VJ (1986) The composite nodule. A structural and functional unit of the reactive human lymph node. Am J Pathol 122:83-91

7. Cesta MF (2006) Normal structure, function, and histology of the spleen. Toxicol Pathol 34:455-465 